Nervenarzt 2021 · 92:57-65

https://doi.org/10.1007/s00115-020-00954-5

Online publiziert: 21 . Juli 2020

(c) Der/die Autor(en) 2020

\author{
Michael Soyka ${ }^{1,2} \cdot$ Susanne Rösner \\ ${ }^{1}$ Medical Park Chiemseeblick, Bernau/Felden, Deutschland \\ ${ }^{2}$ Psychiatrische Universitätsklinik, LMU München, München, Deutschland \\ ${ }^{3}$ Forel Klinik, Ellikon an der Thur, Schweiz
}

\title{
Pharmakotherapie der Alkoholentwöhnung: Update und neue Entwicklungen
}

Therapien auch Harm-reduction-Strategien (Verminderung der Trinkmenge) als mögliche Behandlungsziele definiert.

Als wirksame psychosoziale und psychotherapeutische Behandlungsansätze in der Entwöhnungsbehandlung der Alkoholabhängigkeit sind Interventionskomponenten wie z. B. motivationale Interventionsformen, Verhaltenstherapie und kognitive Verhaltenstherapie wie Kontingenzmanagement, Angehörigenarbeit und Paartherapie mit hohem Empfehlungsgrad genannt. Mitunter wird bei insgesamt moderaten Effekten eine leichte Überlegenheit der kognitiven Verhaltenstherapien gegenüber anderen spezifischen Therapien vermutet, die jedoch in einer aktuellen Metaanalyse [58] nicht bestätigt wurde. Darüber hinaus finden in der S3-Leitlinie die psychotherapeutische Kurzzeittherapie, kognitives Training sowie andere Psychotherapieformen Erwähnung.

Als Medikamente sind Acamprosat, Naltrexon und Disulfiram empfohlen. Für Acamprosat und Naltrexon war bei einer sehr guten Evidenzbasierung (Level 1a) allerdings nur der Empfehlungsgrad B (,sollte gegeben werden“) ausgesprochen worden. Disulfiram erhielt bei Evidenzbasierung $1 \mathrm{~b}$ den Empfehlungsgrad 0. Die Datenlage zur Empfehlung von Nalmefen war bei Erstellung der Leitlinien noch nicht ausreichend. - Tab. 1 gibt einen Überblick über die zur Pharmakotherapie der Alkoholabhängigkeit eingesetzten Substanzen.

Die neurobiologischen und neurochemischen Grundlagen der Alkoholab- hängigkeit sind komplex, werden aber mittlerweile gut verstanden (Übersicht in [95]). Zentrale Strukturen bei der Wirkung von Rauschdrogen sind dopaminerge Neurone im mesolimbischen Bereich (ventrales Tegmentum, Nucleus accumbens) und ihre Projektion in den präfrontalen Kortex, der für Kontrollfunktionen und die Inhibition dysfunktionalen Verhaltens verantwortlich ist. Für Belohnung und Belohnungsantizipation spielt die Ausschüttung von Dopamin im mesolimbischen Bereich eine entscheidende Rolle. Ein abhängiger Konsum von Alkohol entwickelt sich aus einem Zusammenspiel von positiven (z.B. alkoholinduzierte Entspannung, Euphorie) und negativen Konsequenzen (z.B. Entzugssyndrome). Kurz zusammengefasst werden neurochemisch für die positiv verstärkenden Wirkungen vor allem Effekte auf Dopamin, das endogene Opioidsystem, das serotonerge und GABAerge sowie das endogene Cannabinoidsystem verantwortlich gemacht, für die negative Verstärkung vor allem die vermehrte Freisetzung von Kortikotropin-Releasing-Faktor, die GABAerge Down-Regulierung sowie Veränderungen im glutamatergen System (Übersicht in [105]). Aber auch appetitregulierende Hormone wie Ghrelin scheinen von Bedeutung zu sein [27]. Chronischer Alkoholkonsum führt zu erheblichen adaptiven Veränderungen und Anpassungen, vor allem der Rezeptorfunktionen im Gehirn, wobei es beim Alkoholentzug zu einer vermehrten Freisetzung exzitatorischer Neurotransmitter bei erhöh- te Therapieziele wurden in der S3-Leitlinie aus 2016 neben abstinenzorientierten 


\section{Übersichten}

Tab. 1 Übersicht über Medikamente zur Pharmakotherapie der Alkoholabhängigkeit

\begin{tabular}{|c|c|c|c|}
\hline Medikamente & Dosis & Wirkmechanismus & $\begin{array}{l}\text { Andere Indikatio- } \\
\text { nen }\end{array}$ \\
\hline Acamprosat & $1998 \mathrm{mg} / \mathrm{Tag}$ & $\begin{array}{l}\text { Unklar, NMDA-Rezeptor-Agonist, Modulator hyperaktiver glutamaterger Neurone? } \\
\text { Rolle von Kalzium? }\end{array}$ & - \\
\hline Disulfiram & $250-500 \mathrm{mg} / \mathrm{Tag}$ & Inhibition der Acetaldehyddehydrogenase & - \\
\hline Naltrexon & $50 \mathrm{mg} / \mathrm{Tag}$ & $\mu$-Opioid-Rezeptor-Antagonist & Opioidabhängigkeit \\
\hline Nalmefen & $18 \mathrm{mg} / \mathrm{Tag}$ & $\mu$ - und $\delta$-Opioid-Rezeptor-Antagonist, partieller Agonist am к-Opioid-Rezeptor & - \\
\hline Baclofen & $\begin{array}{l}3-80 \mathrm{mg} / \mathrm{Tag}(\mathrm{bis} \\
270 \mathrm{mg})\end{array}$ & $\mathrm{GABA}_{\mathrm{B}}$-Rezeptor-Agonist (metabotrop) & Spastik \\
\hline Gabapentin & $900-1800 \mathrm{mg} / \mathrm{Tag}$ & $\begin{array}{l}\text { Unklar, blockiert spannungsabhängige Kanäle. Keine Wirkung über GABA-Rezepto- } \\
\text { ren }\end{array}$ & $\begin{array}{l}\text { Epilepsie, neuropa- } \\
\text { thischer Schmerz }\end{array}$ \\
\hline Ondansetron & $0,5 \mathrm{mg} / \mathrm{Tag}$ & 5- $\mathrm{HT}_{3}$-Antagonist & $\begin{array}{l}\text { Antiemetikum bei } \\
\text { Krebs (Chemothera- } \\
\text { pie) }\end{array}$ \\
\hline $\begin{array}{l}\text { Prazosin/ } \\
\text { Doxazosin }\end{array}$ & Bis zu $16 \mathrm{mg} / \mathrm{Tag}$ & a1-Rezeptor-Agonist & - \\
\hline Topiramat & Bis $300 \mathrm{mg} / \mathrm{Tag}$ & $\begin{array}{l}\text { Nicht völlig klar, antikonvulsives Medikament, erhöht die GABA } A_{A} \text {-vermittelte neu- } \\
\text { ronale Aktivität und antagonisiert AMPA- und Kainat-Glutamat-Rezeptoren, au- } \\
\text { ßerdem spannungsabhängige Kanäle, schwacher Inhibitor verschiedener anderer } \\
\text { Enzyme }\end{array}$ & $\begin{array}{l}\text { Epilepsie, Migräne, } \\
\text { Lennox-Gastaut- } \\
\text { Syndrom }\end{array}$ \\
\hline Vareniclin & $2 \mathrm{mg} / \mathrm{Tag}$ & Partieller Agonist am nikotinischen a4 $\beta 2$-Acetylcholinrezeptorsubtyp & Rauchen \\
\hline $\begin{array}{l}\text { Y-Hydroxy- } \\
\text { Buttersäure, } \\
\text { GHB }\end{array}$ & - & Präkursor von GABA (schwacher Agonist am $\mathrm{GABA}_{\mathrm{B}}$-Rezeptor) Glutamat, Glycin & Narkolepsie \\
\hline
\end{tabular}

ter Rezeptorempfindlichkeit und einer Übererregbarkeit des Gehirns kommt.

Bislang sind nur wenige Medikamente zur Alkoholentwöhnung zugelassen, obwohl mittlerweile eine ganze Reihe von Substanzen untersucht wurde (Übersicht in [23, 33, 53, 57]). Es gibt etablierte methodische Standards für die Durchführung von Pharmakotherapiestudien bei Alkoholabhängigkeit, wobei primäre Outcome- oder Responderkriterien entweder eine Verbesserung der Abstinenzrate, eine Erhöhung der Zeit bis zum ersten Konsum, eine Verminderung der Rückfallraten oder eine Trinkmengenreduktion sind (siehe [48, 88]). In verschiedenen Therapiestudien werden dabei sehr unterschiedliche Outcomekriterien verwendet $[6,25,40$, 76]. Sekundäre Outcomekriterien sind biologische Marker (Transaminasen, Carbohydrate-defizientes Transferrin [CDT], Ethylglukuronid), sozioökonomische Faktoren („health care utilization") oder Wiederaufnahmen in Kliniken (Übersicht in [99]). Zuletzt wurde von einer Expertengruppe die WHOKlassifikation unterschiedlicher Risikoniveaus („drinking risk levels“) als
Outcomekriterium für Pharmakotherapiestudien empfohlen [26].

Voraussetzung für einen positiven Wirksamkeitsnachweis in klinischen Studien ist unter anderem, dass die pharmakologischen Interventionen auch ausreichend implementiert werden. Hohe Missing- und Drop-out-Raten, wie sie für den Bereich der Alkoholentwöhnung typisch sind, verhindern eine ausreichende Treatment-Implementierung und damit auch die Chancen, in Studien einen tatsächlich vorhandenen Effekt nachweisen zu können [88]. Wichtig ist zudem, dass die Auswahl der Outcomekriterien dem Wirkmechanismus der Substanz angepasst ist.

\section{Substanzen}

\section{Disulfiram}

Viele Jahrzehnte war Disulfiram das einzige zur Behandlung der Alkoholabhängigkeit zugelassene Medikament, welches wegen geringer Verschreibungszahlen in Deutschland nicht mehr vertrieben wird. Disulfiram beeinflusst nicht die biochemischen Effekte von Alkohol, sondern inhibiert das Enzym Aldehyddehydroge- nase, welches das ansonsten schnell verstoffwechselte erste Abbauprodukt von Alkohol, Acetaldehyd, metabolisiert. Toxische erhöhte Acetaldehydspiegel führen zur sogenannten Disulfiram-Alkohol-Reaktion, einer künstlich herbeigeführten Vergiftung mit Übelkeit, Erbrechen, Kreislaufproblemen, Schweißausbrüchen, Hypertension, in schweren Fällen auch kardiovaskulären Reaktionen oder Kollaps (Übersicht in [70, 87]). Die therapeutische Wirkung von Disulfiram basiert primär auf dessen Unverträglichkeit mit Alkohol; dadurch nimmt die Substanz als Aversivtherapeutikum eine Sonderstellung unter den pharmakologischen Therapien der Alkoholabhängigkeit ein. Die klinische Wirkung von Disulfiram basiert auf der Antizipation dieser unerwünschten Effekte. Entscheidend für die therapeutische Wirkung scheint primär die gedankliche Vorwegnahme der Unverträglichkeit mit Alkohol zu sein, nicht die pharmakologische Wirkung der Substanz bzw. das tatsächliche Auftreten einer Alkohol-Disulfiram-Reaktion selbst.

Die größte zur Frage der Wirksamkeit von Disulfiram durchgeführte Untersuchung [30] konnte keinen Wirknach- 
weis erbringen. Die Behandlungsergebnisse bei Supervision der Einnahme von Disulfiram scheinen der nichtsupervidierten Einnahme überlegen zu sein. Eine gute Compliance konnte im Rahmen des Projekts ALITA (Ambulante Langzeit-Intensivtherapie für Alkoholkranke) bei überwachter Einnahme nachgewiesen werden [49].

Wie eine Metaanalyse [92] mit Subgruppenanalyse unter Berücksichtigung des Studiendesigns deutlich macht, zeigt Disulfiram in offenen, nicht aber in randomisierten Doppelblindstudien positive Effekte. Dies ist auf den erwartungsvermittelten Wirkmechanismus der Substanz zurückzuführen: Indem die abstinenzunterstützenden Effekte von Disulfiram im Gegensatz zu anderen pharmakologischen Methoden der Rückfallprophylaxe nicht pharmakologisch, sondern ausschließlich durch Erwartung vermittelt sind, treten die erwartungsinduzierten Therapieeffekte in Studien mit verblindetem Design sowohl in der Interventions- als auch in der Kontrollgruppe auf. Dadurch ist ein Effektnachweis in randomisierten Doppelblindstudien für Disulfiram aus theoretischer Sicht gar nicht möglich [70, 87]. So zeigte sich in einer randomisierten Cross-over-Studie, in der die Erwartung, Disulfiram eingenommen $\mathrm{zu}$ haben, experimentell manipuliert wurde, auch bei placebobehandelten Probanden eine Abnahme der Reizreaktivität („cue reactivity“) auf alkoholassoziierte Reize [91]. Entgegen den methodischen Standards, wie wir sie aus der Wirksamkeitsprüfung anderer Substanzen kennen, sind die Aussagen über die Wirkeffekte zu Disulfiram aus Studien mit offenem Design zuverlässiger [87].

Trotz einer wieder zunehmenden Verordnung von Disulfiram hat die Herstellerfirma Nycomed im Jahr 2011 auf die Erneuerung der Zulassung verzichtet und die Produktion von Disulfiram eingestellt. Der Wirkstoff besitzt in Deutschland derzeit keine Zulassung, kann jedoch auf Basis patientenbezogener Anforderungen über internationale Apotheken bezogen werden. Der spezifische Wirkmechanismus von Disulfiram und die enge Verzahnung von therapeutischem Nutzen und gesundheitlicher

Nervenarzt 2021 · 92:57-65 https://doi.org/10.1007/s00115-020-00954-5

(c) Der/die Autor(en) 2020

\section{Soyka · S. Rösner \\ Pharmakotherapie der Alkoholentwöhnung: Update und neue Entwicklungen}

\section{Zusammenfassung}

Bislang sind nur wenige Medikamente zur pharmakologischen Rückfallprophylaxe der Alkoholabhängigkeit zugelassen. Neben dem in Deutschland nicht mehr vertriebenen Disulfiram sind es die Opioidantagonisten Naltrexon und Nalmefen sowie das vermutlich über glutamaterge Neurone wirkende Acamprosat. Baclofen und $\mathrm{\gamma}$-Hydroxybutyrat (GHB) sind in einzelnen Ländern zugelassen. Wirkstoffe wie z. B. Vareniclin, Gabapentin und Topiramat können für die Rückfallprophylaxe der Alkoholabhängigkeit von Interesse sein, jedoch ist bislang keine Zulassung erfolgt. Vor dem Hintergrund der zur Revision anstehenden S3-Leitlinie zur Diagnose und Behandlung alkoholbezogener Störungen wird der heutige Kenntnisstand zur Pharmakotherapie der Alkoholabhängigkeit dargestellt.

Schlüsselwörter

Alkoholgebrauchsstörungen · Anti-CravingMedikamente $\cdot$ Rückfall $\cdot$ Acamprosat . Naltrexon

\section{Pharmacotherapy of alcohol withdrawal: update and new developments}

\section{Abstract}

So far few medications are approved for prophylactic treatment of alcohol dependence relapse. Apart from disulfiram, which is no longer marketed in Germany, the opioid antagonists naltrexone, nalmefene and the putative glutamate antagonist acamprosate are approved. In some other countries, baclofen and gamma-hydroxybutyrate (GHB) are licensed. Possible other drugs of interest for prophylaxis of alcohol dependence relapse are vareniclin, gabapentin, and topiramate, but so far none of them have received approval. In the light of the currently running revision of the German guidelines for the diagnosis and treatment of alcohol related disorders, an update on the pharmacotherapy of alcohol dependence is presented.

\section{Keywords}

Alcohol use disorders - Anti-Craving-Drugs . Relapse $\cdot$ Acamprosate $\cdot$ Naltrexone
Gefährdung erfordert eine besondere Sorgfaltspflicht bei der Information und Aufklärung des Patienten. So ist in jedem Fall sicherzustellen, dass sich der Patient der Notwendigkeit einer absoluten Abstinenz bewusst ist und eine hohe Abstinenzzuversicht unter Disulfiramtherapie signalisiert. Darüber hinaus sollte die Therapie mit Disulfiram der persönlichen Therapiepräferenz des $\mathrm{Pa}$ tienten entsprechen. Dessen Beteiligung an der Therapieplanung im Sinne einer partizipativen Entscheidungsfindung erweist sich vor dem Hintergrund des Wirkmechanismus von Disulfiram nicht nur im Hinblick auf die Compliance relevant, sondern determiniert darüber hinaus auch das individuelle Risiko einer Therapie mit Disulfiram.

\section{Acamprosat}

Die Substanz ist jetzt seit über 20 Jahren klinisch verfügbar, ihr Wirkmechanismus aber immer noch nicht vollständig geklärt. Acamprosat interagiert nicht mit Alkohol oder anderen Psychopharmaka und hat auch keine psychotropen Effekte, die Substanz wird schlecht resorbiert, sodass bei einem Körpergewicht von über $60 \mathrm{~kg}$ die Einnahme von $6 \times 330 \mathrm{mg}$ (1998 mg) notwendig ist, was mitunter auch zu Compliance-Problemen beitragen kann. Für Acamprosat wird eine Wirkung über glutamaterge Neurone vermutet, auch wurde die Vermutung geäußert, wonach die Wirkung ausschließlich auf das Kalziumatom des Acamprosatmoleküls zurückführen ist [96]. 


\section{Übersichten}

\begin{tabular}{|c|c|c|c|c|c|c|c|}
\hline Autor & Jahr & $\begin{array}{l}\text { RCT (ge- } \\
\text { samt) }\end{array}$ & $N$ (gesamt) & $\begin{array}{l}\text { Konsum - Gesamt- } \\
\text { effekt }\end{array}$ & Konsum - KI & $\begin{array}{l}\text { Rückfall - Gesamtef- } \\
\text { fekt }\end{array}$ & Rückfall - KI \\
\hline Rösner et al. [85] & 2010 & 24 & 6915 & $0,86(\mathrm{RR})$ & 0,81 bis 0,91 & 0,99 (RR) & 0,94 bis 1,04 \\
\hline Maisel et al. [57] & 2013 & 16 & 4349 & 0,36 (Hedges g) & 0,25 bis 0,47 & 0,07 (Hedges g) & $-0,08$ bis $-0,22$ \\
\hline Jonas et al. [43] & 2014 & 16 & 4847 & $-0,09$ (RD) & $-0,14$ bis $-0,04$ & $-0,01(\mathrm{RD})$ & $-0,04$ bis $-0,03$ \\
\hline Donoghue et al. [23] & 2015 & 22 & 5236 & 0,83 (RR) & 0,78 bis 0,89 & - & - \\
\hline
\end{tabular}

\begin{tabular}{|c|c|c|c|c|c|c|c|}
\hline Autor & Jahr & $\begin{array}{l}\text { RCT (ge- } \\
\text { samt) }\end{array}$ & $N$ (gesamt) & $\begin{array}{l}\text { Konsum - Gesamt- } \\
\text { effekt }\end{array}$ & Konsum - KI & $\begin{array}{l}\text { Rückfall - Gesamtef- } \\
\text { fekt }\end{array}$ & Rückfall - KI \\
\hline Rösner et al. & 2010 & 50 & 7793 & 0,96 (RR) & 0,92 bis 1,00 & 0,83 (RR) & 0,76 bis 0,90 \\
\hline Maisel et al. [57] & 2013 & 45 & 5434 & 0,12 (Hedges g) & 0,05 bis 0,18 & 0,19 (Hedges g) & 0,12 bis 0,25 \\
\hline Jonas et al. [43] & 2014 & 19 & 2875 & $-0,05$ (RD) & $-0,10$ bis $-0,002$ & $-0,09$ (RD) & $-0,13$ bis $-0,04$ \\
\hline Donoghue et al. [23] & 2015 & 27 & 4199 & 0,92 (RR) & 0,86 bis 1,00 & 0,85 (RR) & 0,78 bis 0,93 \\
\hline
\end{tabular}

Acamprosat wurde intensiv untersucht. Es gibt mehrere Metaanalysen ([23, 43, 57, 85]; vgl. - Tab. 2). In den beiden größeren Metaanalysen wurden 27 Studien mit über 7000 Patienten eingeschlossen $[43,57]$. Das CochraneReview zu Acamprosat [85] zeigt unter anderem eine signifikante Reduktion des Konsumrisikos auf $81 \%$ des entsprechenden Risikos in der Kontrollgruppe (relatives Risiko $[\mathrm{RR}]=0,86$; 95\%-KI 0,81-0,91). Bei insgesamt heterogenen Behandlungsergebnissen wurde im Vergleich zu Placebo eine Reduktion der Trinktage gezeigt. Acamprosat ist in Europa von der European Medicines Agency und in den USA von der FDA zugelassen worden. Acamprosat wird üblicherweise gut toleriert, die häufigste milde Nebenwirkung von Acamprosat ist Durchfall (weicher Stuhl), der nach einigen Tagen häufig abklingt.

Es gibt zahlreiche placebokontrollierte Doppelblindstudien mit Acamprosat, wenige Vergleichsstudien zu Naltrexon, wobei die größte Untersuchung, die in den USA durchgeführte COMBINE-Studie [9], keinen Wirknachweis von Acamprosat erbrachte. Auch die deutsche Studie [46] zeigte bessere Behandlungsergebnisse für den Opiatantagonisten Naltrexon als für Acamprosat bei gleichzeitigem Vorteil einer kombinierten Behandlung unter Nutzung beider Substanzen.

\section{Opiatantagonisten (Naltrexon,} Nalmefen)

Naltrexon ist ein nichtselektiver Opioidantagonist am $\mu$-, $\kappa$ - und $\delta$-Opioidrezeptor. Zahlreiche pharmakologische Untersuchungen haben gezeigt, dass Naltrexon die subjektiven Effekte von Alkohol vermindern kann, z. B. Alkoholverlangen (Craving), alkoholinduzierte Stimulation, Sedation und auch negative Stimmungen nach Alkoholkonsum [80]. Naltrexon blockiert die positiv verstärkenden „hedonischen“ Effekte von Alkohol, darüber sind über die Beeinflussung des mesolimbischen Dopaminsystems auch motivationale Effekte anzunehmen. Die Interaktionen zwischen Opioidrezeptorblockade und Dopaminausschüttung sind komplex und dynamisch, gesichert ist aber, dass durch die Blockade des Opioidendorphinsystems indirekt auch die Ausschüttung von Dopamin im Nucleus accumbens reguliert wird (Übersicht in [37]).

In Untersuchungen wurde üblicherweise eine Dosis von 50 mg ( 1 Tbl. oral) untersucht. Die ersten Untersuchungen wurden von O'Malley et al. [73] und Volpicelli et al. [104] durchgeführt, zahlreiche andere placebokontrollierte Doppelblindstudien haben sich angeschlossen. Metaanalysen haben gezeigt, dass Naltrexon vor allem die Trinkmenge bzw. die Rückfallrate zu schwerem Trinken vermindert ([23, 43, 57, 86]; vgl. • Tab. 3) und weniger Einfluss auf die Abstinenzrate hat. Das Cochrane-Review zu Naltrexon [86] zeigt eine signifikante Reduktion des Risikos für einen Rückfall (= Konsum von 5 „standard drink units“ oder mehr) auf $83 \%$ des Risikos in der Kontrollgruppe $(R R=0,83 ; 95 \%$-KI 0,76-0,90). Eine Depotform von Naltrexon ist in den USA, nicht aber in Deutschland zur Behandlung der Alkoholabhängigkeit zugelassen [32].

Basierend auf dem Datensatz der Cochrane-Analyse [86] wurde vor kurzem eine Metaanalyse über 38 Studien mit 11.194 Teilnehmern publiziert [13], die eine Reihe von Nebenwirkungen identifizierte, die bei Naltrexon häufiger sind als bei Placebo (verminderter Appetit, Schläfrigkeit, Übelkeit, Benommenheit, Schwitzen und Erbrechen). Bei Opiatabhängigkeit führt die Gabe von Naltrexon zur Auslösung eines Opioidentzugssyndroms.

Nalmefen hat bis auf ein Atom eine ähnliche Struktur wie Naltrexon, aber ein etwas anderes Rezeptorprofil (• Abb. 1). Nalmefen ist wie Naltrexon ein Antagonist am $\mu$-Opioidrezeptor und Modulator am $\delta$-Opioidrezeptorsubtyp. Die Wirkung von Nalmefen wird darüber hinaus über den $\kappa$-Rezeptor vermittelt, wo sich partiell agonistische und antagonistische Wirkungen zeigen [94].

Basierend auf einer Reihe von kleineren Studien wurde Nalmefen in den vergangenen Jahren ausschließlich zur 

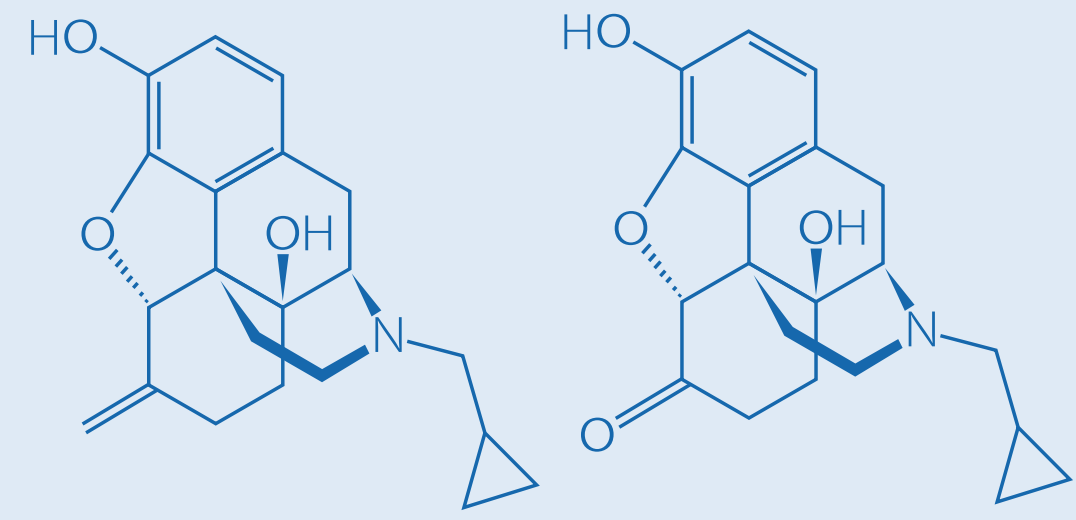

Nalmefen
Naltrexon

Abb. 1 ॥ Strukturformeln von Nalmefen und Naltrexon

Trinkmengenreduktion eingesetzt. In den letzten Jahren wurde die Wirksamkeit von Nalmefen zur Trinkmengenreduktion in drei größeren placebokontrollierten Untersuchungen zur Trinkmengenreduktion unter Verwendung eines „As-needed“-Ansatzes geprüft [35, $60,100,101]$. Dabei wird Nalmefen bei Bedarf, also zum Beispiel in Situationen eines erhöhten Cravings oder anderer Risikofaktoren, eingesetzt. Die Untersuchungen zeigen insgesamt eine mäßige Reduktion der Trinkmenge. Allerdings ist zu berücksichtigen, dass in die Studien nur Patienten mit leichterem und moderatem Schweregrad einer Alkoholabhängigkeit eingeschlossen wurden.

2013 wurde die Substanz von der European Medicines Agency zugelassen, in den USA ist Nalmefen nicht verfügbar. Auch wenn Harm-reduction-Strategien bei Alkoholabhängigkeit prinzipiell als sinnvoll zu bewerten sind [28, 72, 98], müssen die Ergebnisse auch vor dem Hintergrund methodischer Aspekte interpretiert werden. So ergeben sich in einer Metaanalyse mit 5 randomisierten, kontrollierten Studien zu Nalmefen [75] Hinweise auf selektive Drop-out-Raten sowie eine mangelnde Repräsentativität der Stichprobe. So liegt die durchschnittliche Trinkmenge der Probanden unter den üblichen Durchschnittswerten alkoholabhängiger Samples.

Positive Ergebnisse wurden aber vor Kurzem von einer weiteren Arbeitsgrup- pe publiziert [66], die Nalmefen 10 und $20 \mathrm{mg}$ gegen Placebo in einer großen Studie über 24 Wochen bei alkoholabhängigen Patienten untersuchte und dabei erneut eine Reduktion des „heavy drinking“ im Vergleich zu Placebo fand. Auch offene Studien mit Nalmefen haben einen positiven Effekt gezeigt [16]. Der „Asneeded“-Ansatz, vor allem bei weniger schwer betroffenen Patienten mit Alkoholkonsumstörungen, ist ein interessanter, den es weiter zu untersuchen gilt.

Eine Reihe von anderen Substanzen habt ein Potenzial im Hinblick auf die pharmakologische Rückfallprophylaxe bei Alkoholabhängigkeit, wobei die wichtigsten Medikamente aus anderen Indikationsbereichen stammen und die neurochemischen Effekte extrem unterschiedlich sind - was unterstreicht, dass die neurobiologische Alkoholforschung bislang keine „magic bullets“ entwickelt hat (•Tab. 1).

\section{Baclofen}

Baclofen ist ein selektiver $\mathrm{GABA}_{\mathrm{B}}$-Rezeptor-Agonist, der als Arzneistoff aus der Gruppe der Muskelrelaxanzien zur Behandlung von Spastizität und bei multipler Sklerose eingesetzt wird. Das Interesse ist nach einem Eigenbericht eines mittlerweile verstorbenen französischen Arztes, der seine „Heilung“ vom Alkoholismus auf eine Hochdosistherapie mit Baclofen bis $250 \mathrm{mg} / \mathrm{Tag}$ zurück- führte [7], gestiegen. Baclofen wird rasch resorbiert.

Eine Reihe von randomisierten Therapiestudien mit verschiedenen Dosierungen und auch unterschiedlichen Ergebnissen wurde durchgeführt [1-3, 31, 67]. Die Ergebnisse der US-amerikanischen Studie [31], der australischen [67], der holländischen [11] und der israelischen Studien [78] konnten keine Überlegenheit gegenüber Placebo nachweisen. In der BacALD-Studie war Baclofen ein wirksames Medikament, höhere Dosen waren mit schwereren Nebenwirkungen assoziiert [68]. Eine deutsche Untersuchung [69] mit Baclofen bis $270 \mathrm{mg} / \mathrm{Tag}$ zeigte deutlich positive Effekte auf die Abstinenzraten.

Baclofen ist in Frankreich zugelassen, auch wenn das Sicherheitsprofil der Substanz kritisch diskutiert wird [84, 90]. Nebenwirkungen sind Müdigkeit, Schlafstörungen, Übelkeit und Benommenheit. Vor allem die Hochdosisgabe erscheint problematisch [17] und ist für eine höhere Rate von Hospitalisierungen und Todesfällen verantwortlich. Aufgrund alkoholähnlicher Wirkungen von Baclofen im Gehirn wird diskutiert, inwieweit die Therapie mit Baclofen als Substitutionsbehandlung bei Alkoholismus bezeichnet werden kann [20]. In Frankreich wurden zwei sehr große Untersuchungen durchgeführt. In der ALPADIR-Studie [82] konnte keine Überlegenheit von Baclofen gegenüber Placebo nachgewiesen werden, in der BACLOVILLE-Studie [41] zeigte sich ein signifikanter Effekt in der Baclofengruppe für Abstinenz und die Reduktion der WHO-Risikoniveaus.

In einer Metaanalyse mit 14 randomisierten klinischen Studien wurden keine signifikanten Effekte von Baclofen nachgewiesen, dies ebenso in einer Post-hocAnalyse der Studien mit höheren Dosen [15]. Eine aktuelle Cochrane-Analyse [65] mit insgesamt 12 randomisierten klinischen Studien konnte die Wirksamkeit von Baclofen ebenfalls nicht bestätigen. Allerdings wurde auch eine starke Heterogenität der Effektstärken deutlich, die für den Einfluss von Stichproben und Designmerkmalen spricht. Die in einigen Studien mit hoher Studienqualität nachgewiesenen positiven Effekte (z. B. [69]) sprechen weiter für eine mögliche Wirk- 
samkeit von Baclofen. In weiteren Studien ist zu prüfen, inwieweit bestimmte Merkmale der Behandlung (z. B. individuelle Dosisanpassung) die Wirksamkeit von Baclofen beeinflussen.

\section{Antikonvulsiva (Gabapentin, Pregabalin, Topiramat)}

Gabapentin ist als Antiepileptikum und zur Behandlung der Neuralgie und des Restless-legs-Syndroms zugelassen (Dosierungen 300 bis $1800 \mathrm{mg} / \mathrm{Tag}$ ). Die American Psychiatric Association (APA) empfiehlt Gabapentin und das unten besprochene Topiramat bei Unverträglichkeit von Acamprosat und Naltrexon oder bei fehlendem therapeutischem Ansprechen auf die gängigen Anti-Craving-Medikamente. Der Wirkmechanismus von Gabapentin ist nicht völlig klar, es beeinflusst spannungsabhängige Ca-Kanäle [62] und verstärkt die GABAerge Aktivität, bindet aber nicht an GABA-Rezeptoren.

Eine kürzlich erschienene Metaanalyse [52] mit 7 randomisierten, kontrollierten Therapiestudien zu Gabapentin fand einen mittelgradigen Effekt auf die Anzahl der "heavy drinking days“, alle anderen Outcomekriterien waren nicht signifikant. Eine weitere Metaanalyse [5] konnte auf der Grundlage von 10 Studien einen moderaten Effekt auf die Symptome eines Alkoholentzugs und auf das Alkoholcraving nachweisen. Pharmacovigilance-Meldungen des FDA Adverse Events Reporting System finden Hinweise auf ein Missbrauchspotenzial der Substanz [103], das vermutlich auf den GABAergen Wirkmechanismus von Gabapentin und seine entspannenden Effekte zurückzuführen ist.

Für Pregabalin liegen nur einige wenige Befunde für die Behandlung des Alkoholentzugssyndroms vor [29], nichts zur Rückfallprophylaxe. Ohnehin wäre diese Substanz wegen ihres Suchtpotenzials als Anti-Craving-Medikament kritisch zu bewerten.

Topiramat ist ein Antiepileptikum, dass am GABA-Rezeptor wirkt und gleichzeitig auch die Aktivität glutamaterger Rezeptorsubtypen reduziert [50, 51]. Es gibt eine Reihe randomisierter Studien zur Wirksamkeit von Topira- mat, meist wurden Dosen von 200 bis 300 mg eingesetzt. Wie Metaanalysen zeigen, sind die therapeutischen Effekte mit denen etablierter Anti-Craving-Substanzen vergleichbar, die Datenlage ist jedoch nicht ausreichend, um aussagekräftige Schlussfolgerungen abzuleiten (z. B. [12, 77]). Als häufigste Nebenwirkung zeigen sich unter anderem Schwindel, Müdigkeit, Parästhesien und Appetitverlust, welche hinsichtlich Verträglichkeit und Compliance problematisch sind.

\section{Ketamin}

Zu den interessanten neuen Ansätzen gehört der nichtkompetitive glutamaterge NMDA-Rezeptor-Antagonist Ketamin [18]. Hier konnte experimentell gezeigt werden, dass Ketamin verstärkende Effekte von Alkohol vermindern kann [19]. Kontrollierte klinische Studien stehen noch aus.

\section{Vareniclin}

Vareniclin ist ein partieller Agonist am a4ß2-Nikotin-Rezeptor und ein voller Agonist an nikotinischen Acetylcholinrezeptoren (nAChR) und für die Behandlung von Nikotinabhängigkeit zugelassen. Es gibt auch einige wenige Untersuchungen zur Wirksamkeit bei alkoholabhängigen Patienten [54, 56], darunter auch Untersuchungen mit negativem Ergebnis [21]. Der Einsatz bei komorbiden Rauchern ist naheliegend. Aktuell laufen einige Studien, auch Vergleichsuntersuchungen zu Naltrexon [54]. Eine aktuelle Metaanalyse mit über 9 placebokontrollierten Doppelblindstudien $(N=585$, Behandlungsdauer 4-13 Wochen) zeigt, dass Vareniclin im Vergleich zu Placebo nicht die Zahl der „heavy drinking days", aber die Menge des konsumierten Alkohols insgesamt vermindern konnte [74].

\section{Y-Hydroxybutyrat (GHB)}

Kontrovers wird der Einsatz von „sodium oxybate" (SMO) und $\gamma$-Hydroxybutyrat (GHB), einer über den GABA-Rezeptor wirkenden stark psychotropen Substanz, bewertet, die in Österreich und in Italien zur Behandlung von Alkoholentzugssyn- dromen und Alkoholabhängigkeit zugelassen ist (Übersicht in [44]). GHB hat selber ein starkes Suchtpotenzial und traurige Berühmtheit als „rape drug“ erlangt, als Substanz, die im Rahmen von Straftaten genutzt wird und Amnesie hervorruft, sodass sich die Opfer nicht mehr an die Tat oder den Tathergang erinnern können. Eine Metaanalyse [14] zeigt unter Einschluss von 7 randomisierten, kontrollierten Studien eine signifikante Überlegenheit von GHB gegenüber Placebo für verschiedene Outcomes der Alkoholentwöhnung, im niedrigen Dosisbereich scheint die Substanz zudem gut verträglich zu sein [44]. Dem Wirksamkeitsnachweis steht das vergleichsweise hohe Missbrauchspotenzial von GHB gegenüber, welches die Eignung der Substanz als pharmakologische Unterstützung der Alkoholentwöhnung stark einschränkt. Zusätzlich problematisch für die Verwendung der Substanz im Bereich der Alkoholtherapie ist die potenzierende Wirkung von GHB auf Alkohol (Übersicht in [102]).

\section{Antidepressiva und Antipsychotika}

Konventionelle psychotrope Substanzen wie z. B. Antidepressiva oder Antipsychotika, die das Dopaminsystem blockieren [47], haben sich in der pharmakologischen Rückfallprophylaxe zumindest bei nicht psychisch kranken Alkoholabhängigen als nicht wirksam erwiesen (Übersicht in [93]).

\section{Weitere Substanzen}

Andere Untersuchungen, bei denen verschiedene Substanzen eingesetzt worden sind, umfassen Ondansetron [42, 45], den a1-Rezeptor-Blocker Prazosin und Doxazin [36], den Glukuronidrezeptorblocker Mifepriston, außerdem Oxytozin und weitere Substanzen [105]. Für Prazosin liegen einige Untersuchungen vor [54], die letzte von Simpson et al. [89], die 92 Patienten mit $16 \mathrm{mg}$ Prazosin oder Placebo behandelte und einen gewissen klinischen Effekt auf die Trinkmenge beschreiben konnte. Als Nebenwirkung tritt Benommenheit relativ häufig auf. 


\section{Pharmakogenetik}

Hier liegen bislang nur relativ wenig neue Befunde vor (Übersicht in [38, 39, $79,83]$ ), was angesichts der bislang begrenzten Auswahl pharmakotherapeutischer Optionen nicht überrascht. Am ehesten auch von klinischem Interesse waren bislang funktionelle Polymorphismen im OPRM1- und OPRK1-Gen, die wahrscheinlich die Effekte von Opiatantagonisten wie Naltrexon modifizieren. Dazu liegt inzwischen eine Reihe experimenteller und klinischer Befunde vor [79, 83]. Eine aktuelle Metaanalyse mit 7 randomisierten, kontrollierten Studien fand einen moderaten Effekt des Asn40Asp-SNP für das Outcome „drinks per day“, ansonsten keine weiteren Hinweise, dass das rs1799971-GAllel im OPRM1-Gen einen Einfluss auf das Ansprechen auf eine Therapie mit Naltrexon hat [39]. Für Acamprosat könnten Variationen in den glutamatergen Rezeptoren (GATA4, GREN2b) von Bedeutung sein, für Topiramat Polymorphismen in den glutamatergen AMPAund Kainatrezeptoren (GRIK1 und 2).

Eine Übersichtsarbeit über den Einfluss serotonerger Genvariationen auf das Ansprechen pharmakotherapeutischer Methoden der Rückfallprophylaxe bei Abhängigkeitserkrankungen weist auf die Bedeutung von Genen für das Enzym Tryptophanhydroxylase 2 (TPH2) und den Serotonintransporter (SLC6A4) hin, welche die Wirkung von Ondansetron und Disulfiram modulieren [10].

In der BacALD-Studie wurde für Baclofen die Relevanz des RS29220-SNP im $\mathrm{GABA}_{\mathrm{B}}$-Rezeptor für den Therapieerfolg und möglicherweise auch für Unverträglichkeitsreaktionen für Baclofen gezeigt [68].

\section{Schlussfolgerung}

Bislang sind nur wenige Medikamente zur Pharmakotherapie der Alkoholabhängigkeit zugelassen, einige andere Substanzen, die überwiegend aus anderen medizinischen Indikationsbereichen stammen, wie Baclofen, Topiramat, Vareniclin oder Gabapentin, könnten künftig eine größere Rolle spielen. Die neurochemischen Effekte und Grundlagen der einzelnen Substanzen unterscheiden sich erheblich. Lückenhaft sind die klinischen Daten insbesondere auch bezüglich der Wirksamkeit von Anti-Craving-Substanzen bei komorbider psychischer Störung.

Klinisch von Bedeutung ist aber, dass bereits die bislang verfügbaren Substanzen kaum eingesetzt werden, stattdessen werden häufig konventionelle Psychopharmaka (ohne Wirkungsnachweis in diesem Bereich) eingesetzt, namentlich Antidepressiva, obwohl sie zur Trinkmengenreduktion unwirksam sind [4]. Somit wird eine bereits verfügbare pharmakotherapeutische Option zur Rückfallprophylaxe bei Alkoholabhängigen nicht genützt, man könnte auch sagen „verschenkt“. Stellt man die Verordnungszahlen in Relation zu der hohen Zahl der von Alkoholabhängigkeit betroffenen Personen, kann hier von einer deutlichen Unterversorgung abhängiger Patienten mit pharmakologischen Interventionen gesprochen werden [97]. Diese sind natürlich stets als adjuvante Strategie zur Begleitung und Ergänzung psychotherapeutischer Methoden zu verstehen.

Die immer noch zögerliche Verordnung von Anti-Craving-Substanzen ist durch die Datenlage kaum begründbar. So werden mit Anti-Craving-Substanzen Effekte erzielt, die in ihrer Ausprägung durchaus mit etablierten Therapien anderer psychiatrischer und somatischer Behandlungsbereiche vergleichbar sind (vgl. z. B. [55]). Hinzu kommt, dass die tatsächlichen Effekte der AntiCraving-Behandlung aufgrund methodischer Besonderheiten im Bereich der Abhängigkeitstherapie durch klinische Studien eher unterschätzt als überschätzt werden dürften [88].

An der zurückhaltenden therapeutischen Nutzung von Anti-Craving-Substanzen dürften vermutlich auch psychologische Faktoren wie eine geringe Erfolgserwartung oder die Befürchtung einer eventuellen "Suchtverlagerung“ beteiligt sein. Die Prüfung eigener Vorbehalte, das offene Ansprechen etwaiger Bedenken des Patienten und dessen Beteiligung an einer gemeinsamen Entscheidungsfindung sind im Bereich der Anti-Craving-Behandlung von besonderer Bedeutung.
Auch wenn sich die Basis der klinisch wirksamen Substanzen nur zaghaft zu verbreitern scheint, kann eine zunehmende Anpassung der Strategien an die individuellen Bedürfnisse und Präferenzen des Patienten eine Zunahme der Compliance erzielen. Damit sollte auch das klinische und wissenschaftliche Interesse an den Substanzen wieder stärker in den Fokus rücken.

\section{Korrespondenzadresse}

\section{Prof. Dr. Michael Soyka}

Medical Park Chiemseeblick

Rasthausstraße 25, 83233 Bernau/Felden,

Deutschland

Michael.soyka@med.uni-muenchen.de

Funding. Open Access funding provided by Projekt DEAL.

\section{Einhaltung ethischer Richtlinien}

Interessenkonflikt. M. Soyka war für Amomed, Camurus, Lundbeck und Indiviorals als Berater tätig oder hat Forschungsunterstützung oder Referentenhonorare erhalten. S. Rösner gibt an, dass kein Interessenkonflikt besteht.

Für diesen Beitrag wurden von den Autoren keine Studien an Menschen oder Tieren durchgeführt. Für die aufgeführten Studien gelten die jeweils dort angegebenen ethischen Richtlinien.

Open Access. Dieser Artikel wird unter der Creative Commons Namensnennung 4.0 International Lizenz veröffentlicht, welche die Nutzung, Vervielfältigung, Bearbeitung, Verbreitung und Wiedergabe in jeglichem Medium und Format erlaubt, sofern Sie den/die ursprünglichen Autor(en) und die Quelle ordnungsgemäß nennen, einen Link zur Creative Commons Lizenz beifügen und angeben, ob Änderungen vorgenommen wurden.

Die in diesem Artikel enthaltenen Bilder und sonstiges Drittmaterial unterliegen ebenfalls der genannten Creative Commons Lizenz, sofern sich aus der Abbildungslegende nichts anderes ergibt. Sofern das betreffende Material nicht unter der genannten Creative Commons Lizenz steht und die betreffende Handlung nicht nach gesetzlichen Vorschriften erlaubt ist, ist für die oben aufgeführten Weiterverwendungen des Materials die Einwilligung des jeweiligen Rechteinhabers einzuholen.

Weitere Details zur Lizenz entnehmen Sie bitte der Lizenzinformation auf http://creativecommons.org/ licenses/by/4.0/deed.de.

\section{Literatur}

1. Addolorato G, Caputo F, Capristo E et al (2002) Baclofen efficacy in reducing alcohol craving and intake: a preliminary double-blind study 
randomized controlled study. Alcohol Alcohol 37:123-135

2. Addolorato G, Leggio L, Ferulli A et al (2007) Effectiveness and safety of baclofen for maintenance of alcohol abstinence in alcohol-dependent patients with liver cirrhosis: randomized, double-blind controlled study. Lancet 370:1915-1922

3. Addolorato G, Leggio L, Ferrulliu A et al (2011) Dose-response effect of baclofen in reducing daily alcohol intake in alcohol dependence: secondary analysis of a randomized, double-blind, placebocontrolled trial. Alcohol Alcohol 46:312-317

4. Agabio R, Trogu E, Pani PP (2018) Antidepressants for the treatment of people with co-occurring depression and alcohol dependence. Cochrane Database Syst Rev 4(4):CD8581

5. Ahmed S, Stanciu CN, Kotapati PV (2019) Effectiveness of gabapentin in reducing cravings and withdrawal in alcohol use disorder: a metaanalytic review. Prim Care Companion CNS Disord 21(4):19r02465

6. Allen JP (2003) Measuring outcome in interventions for alcohol dependence and problem drinking: executive summary of a conference sponsored by the national institute on alcohol abuse and alcoholism. Alcohol Clin Exp Res 27(10):1657-1660

7. Ameisen 0 (2005) Complete and prolonged suppression of symptoms and consequences of alcohol-dependence using high-dose baclofen: a self-case report of a physician. Alcohol Alcohol 40:147-150

8. American Psychiatric Association (2013) Diagnostische und Statistische Manual Psychischer Störungen, 5. Aufl. American Psychiatric Publishing, Arlington, VA

9. Anton RF, O'Malley SS, Ciraulo DA et al (2006) Combined pharmacotherapies and behavioral interventions for alcohol dependence: the COMBINE study: a randomized controlled trial. JAMA 295(17):2003-2017

10. Bauer IE, Graham DP, Soares JC et al (2015) Serotonergic gene variation in substance use pharmacotherapy: a systematic review. Pharmacogenomics 16(11):1307-1314

11. Beraha EM, Salemink E, Goudriaan AE et al (2016) Efficacy and safety of high-dose baclofen for the treatment of alcohol dependence: a multicentre, randomised, double-blind controlled trial. Eur Neuropsychopharmacol 26(12):1950-1959

12. Blodgett JC, Del Re AC, Maisel NC et al (2014) A meta-analysis of topiramate's effects for individuals with alcohol use disorders. Alcohol Clin ExpRes 38:1481-1488

13. Bolton M, Hodkinson A, Boda Set al (2019) Serious adverse events reported in placebo randomised controlled trials of oral naltrexone: a systematic review and meta-analysis. BMC Med 17(1):10

14. Brambilla R, Vigna-Taglianti F, Avanzi G et al (2012) II gamma-idrossibutirrato (GHB) nel trattamento a medio/lungo termine della dipendenza da alcol: una revisione sistematica [gamma-hydroxybutyrate (GHB) for mid/long term treatment of alcohol dependence: a systematic review. Riv Psichiatr 47(4):269-280

15. Bschor T, Henssler J, Müller M et al (2018) Baclofen for alcohol use disorder - a systematic metaanalysis. Acta Psychiatr Scand 138(3):232-242

16. Castera P, Stewart E, Großkopf J et al (2018) Nalmefene, given as needed, in the routine treatment of patients with alcohol dependence: an interventional, open-label study in primary care. Eur Addict Res 24(6):293-303
17. Chaignot C, Zureik M, Rey G et al (2018) Risk of hospitalization and death related to baclofen for alcohol use disorders: comparison with nalmefene, acamprosate, and naltrexone in a cohort study of 165334 patients between 2009 and 2015 in France. Pharmacoepidemiol Drug Saf 27:1239-1248

18. Crowley NA, Magee SN, Feng M et al (2019) Ketamine normalizes binge drinking-induced defects in glutamatergic synaptic transmission and ethanol drinking behavior in female but not male rats. Neuropharmacology 149:35-44

19. Das RK, Gale G, Walsh K et al (2019) Ketamine can reduce harmful drinking by pharmacologically rewriting drinking memories. Nat Commun 10(1):5187

20. de Beaurepaire $R$ (2018) A review of the potential mechanisms of action of baclofen in alcohol use disorder. Front Psychiatry 9:506

21. de Bejczy A, Lof E, Walther L et al (2015) Varenicline for treatment of alcohol dependence: a randomized, placebo-controlled trial. Alcohol Clin ExpRes 39:2189-2199

22. Dilling $H$, Mombour W, Schmidt M (2015) Internationale Klassifikation psychischer Störungen, 10. Aufl. Huber, Bern (ICD-10 Kapitel V (F))

23. Donoghue K, Elzerbi C, Saunders R (2015) The efficacy of acamprosate and naltrexone in the treatment of alcohol dependence, Europe versus the rest of the world: a meta-analysis. Addiction 110(6):920-930

24. Edwards G, Brown D, Oppenheimer E et al (1988) Long term outcome for patients. With drinking problems: the search for predictors. Br J Addict 83:917-927

25. European Medicines Agency (2010) Guideline on the development of medicinal products for the treatment of alcohol dependence. European Medicines Agency, London

26. Falk DE, O'Malley SS, Witkiewitz K et al (2019) Alcohol clinical trials initiative (ACTIVE) workgroup. Evaluation of drinking risk levels as outcomes in alcohol pharmacotherapy trials: a secondary analysis of 3 randomized clinical trials. JAMA Psychiatry 76(4):374-381

27. Farokhnia M, Faulkner ML, Piacentino Det al (2019) From a gut hormone to a potential therapeutic target for alcohol use disorder. Physiol Behav 204:49-57

28. Fitzgerald N, Angus K, Elders A et al (2016) Weak evidence on nalmefene creates dilemmas for clinicians and poses questions for regulators and researchers. Addiction 111:14477-11487

29. Freynhagen R, Backonja M, Schug S et al (2016) Pregabalin for the treatment of drug and alcohol withdrawal symptoms: a comprehensive review. CNSDrugs 30:1191-1200

30. Fuller RK, Branchey L, Brightwell DR et al (1986) Disulfiram treatment of alcoholism. A veterans administration cooperative study. JAMA 256(11):1449-1455

31. Garbutt JC, Kampov-Polevoy AB, Gallop P (2010) Efficacy and safety of baclofen for alcohol dependence: a randomized, double-blind, placebocontrolled trial. Alcohol Clin Exp Res 34:1849-1857

32. Garbutt JC, Kranzler HR, O'Malley SS, Vivitrex Study Group et al (2005) Efficacy and tolerability of long-acting injectable naltrexone for alcohol dependence: a randomized controlled trial. JAMA 293(13):1617-1625

33. Goh ET, Morgan MY (2017) Review article: pharmacotherapy for alcohol dependence-the why, the what and the wherefore. Aliment Pharmacol Ther 45:865-882
34. Grant BF, Goldstein RB, Saha TD et al (2015) Epidemiology of DSM-5 alcohol use disorder: results from the national epidemiologic survey on alcohol and related conditions III. JAMA Psychiatry 72(8):757-766

35. Gual A, He Y, Torup L et al (2013) A randomized, double-blind, placebo-controlled, efficacy study of nalmefene, as-needed use, in patients with alcohol dependence. Eur Neuropsychopharmacol 23:1432-1442

36. Haass-Koffler CL, Swift RM (2018) Noradrenergic targets for the treatment of alcohol use disorder. Psychopharmacology 235:1625-1634

37. Hansson AC, Gründer G, Hirth N et al (2019) Dopamine and opioid systems adaptation in alcoholism revisited: convergent evidence from positron emission tomography and postmortem studies. Neurosci Biobehav Rev 106:141-164

38. Hartwell EE, Kranzler KR (2019) Pharmacogenetics of alcohol use disorder treatments: an update. Expert Opin Drug Metab Toxicol 15:553-564

39. Hartwell EE, Feinn R, Morris PE (2020) Systematic review and meta-analysis of the moderating effect of rs1799971 in OPRM1, the Mu-opioid receptor gene, on response to naltrexone treatment of alcoholuse disorder. Addiction. https://doi.org/10. 1111/add.14975

40. Hasin DS, Wall M, Witkiewitz Ket al (2018) Change in non-abstinent, WHO drinking risk levels and alcohol dependence, $A 3$ year follow-up results in the US general polulation. Alcohol Clin Exp Res 42:2256-2265

41. Jaury P (2016) Bacloville: clinical efficacy study of high dose baclofen in reducing alcohol consumption in high risk drinkers. Berlin ISBRA Congress, Berlin

42. Johnson BA, Ait-Daoud C, Seneviratne J et al (2011) Pharmacogenetic approach at the serotonin transporter gene as a method of reducing the severity of alcohol drinking. Am J Psychiatry 168:265275

43. Jonas DE, Amick HR, Feltner C et al (2014) Pharmacotherapy for adults with alcohol use disorders in outpatient settings: a systematic review and metaanalysis. JAMA 311(18):1889-1900

44. Keating GM (2014) Sodium oxybate: a review of its use in alcohol withdrawal syndrome and in the maintenance of abstinence in alcohol dependence. Clin Drug Investig 34:63-80

45. Kenna GA, Zywiak WH, Swift RM et al (2014) Ondansetron reduces naturalistic drinking in nontreatment-seeking alcohol-dependent individuals with the LL 5'-HTTLPR genotype: a laboratory study. Alcohol Clin Exp Res 38:1567-1574

46. Kiefer F, Jahn H, Tarnaske T et al (2003) Comparing and combining naltrexone and acamprosate in relapse prevention of alcoholism: a double-blind, placebo-controlled study. Arch Gen Psychiatry 60(1):92-99

47. Kishi T,DevyS, Chekuri Retal (2013) Antipsychotics for primary alcohol dependence: a systematic review and meta-analysis of placebo-controlled trials. JClin Psychiatry 74(7):e642-54

48. Klemperer EM, Hughes JR, Naud S (2018) Study characteristics influence the efficacy of substance abuse treatments: a meta-analysis of medications for alcohol use disorder. Drug Alcohol Depend 190:229-234

49. Krampe H, Spies CD, Ehrenreich H (2011) Supervised disulfiram in the treatment of alcohol use disorder: a commentary. Alcohol Clin Exp Res 35(10):1732-1736

50. Kranzler HR, Covault J, Feinn R et al (2014a) Topiramate treatment for heavy drinkers: mode- 
ration by a GRIK1 polymorphism. Am J Psychiatry 171:445-452

51. Kranzler HR, Armeli S, Feinn R et al (2014b) GRIK1 genotype moderates topiramate's effects on daily drinking level, expectations of alcohols positive effects and desire to drink. Int J Neuropsychopharmacol 17:1549-1556

52. Kranzler HR, Feinn R, Morris P et al (2019) A metaanalysis of the efficacy of gabapentin for treating alcohol use disorder. Addiction 114:1547-1555

53. Kranzler HR, Soyka M (2018) Diagnosis and pharmacotherapy of alcohol use disorder. A review. JAMA 320:815-826

54. Leggio L, Falk DE, Ryan ML (2019) Medication development for alcohol use disorder: a focus on clinical studies. Handb Exp Pharmacol. https://doi. org/10.1007/164_2019_295

55. Leucht S, Hierl S, Kissling W (2012) Putting the efficacy of psychiatric and general medicine medication into perspective: review of metaanalyses. Br JPsychiatry 200(2):97-106

56. Litten RZ, Ryan ML, Fertig JR et al (2013) A double-blind, placebo-controlled trial assessing the efficacy of varenicline tartrate for alcohol dependence. J Addict Med 7:277-286

57. Maisel NC, Blodgett JC, Wilbourne PL et al (2013) Meta-analysis of naltrexone and acamprosate for treating alcohol use disoorders: when are these medications most helpful? Addiction 108(2):275-293

58. Magill M, Ray L, KilukB et al (2019) A meta-analysis of cognitive-behavioral therapy for alcohol or other drug use disorders: treatment efficacy by contrast condition. JConsult Clin Psychol 87:10931105

59. Mann K, Hoch E, Batra A (2016) S3-Leitlinie Screening, Diagnose und Behandlung alkoholbezogener Störungen. Springer, Heidelberg, Berlin

60. Mann K, Bladström A, Torup Letal (2013) Extending the treatment options in alcohol dependence: a randomized controlled study of as-needed nalmefene. Biol Psychiatry 73:706-713

61. Marshall EJ, Edwards G, Taylor C (1994) Mortality in men with drinking problems: a 20-year follow-up. Addiction 89:1293-1298

62. Mason BJ, Quello S, Shadan F (2018) Gabapentin for the treatment of alcohol use disorder. Expert Opin Investig Drugs 27:113-124

63. McCrady BS, Owens MD, Borders AZ (2014) Psychosocial approaches to alcohol use disorders since 1940: a review. J Stud Alcohol Drugs Suppl 75(17):68-78

64. Miller WR, Wilbourne PL (2002) Mesa grande: a methodological analysis of clinical trials of treatments for alcohol use disorders. Addiction 27:867-886

65. Minozzi S, Saulle R, Rösner S (2018) Baclofen for alcohol use disorder. Cochrane Database Syst Rev 11:CD12557

66. Miyata $H$, Takahashi M, Murai $Y$ et al (2019) Nalmefene in alcohol-dependent patients with a high drinking risk: randomized controlled trial. Psychiatry Clin Neurosci 73:697-706

67. Morley KC, Baillie A, Leung S et al (2014) Baclofen for the treatment of alcohol dependence and possible role of comorbid anxiety. Alcohol Alcohol 49:654-660

68. Morley KC, Luquin N, Baillie A (2018) Moderation of baclofen response by a GABAB receptor polymorphism: results from the BaCALD randomized controlled trial. Addiction 113(12):2205-2213

69. Müller CA, Geisel O, Pelz P et al (2015) High-dose baclofen for the treatment of alcohol dependence (BACLADstudy): a randomized, placebo-controlled trial. Eur Neuropsychopharmacol 25:1167-1177
70. Mutschler J, Grosshans M, Soyka M (2016) Current findings and mechanisms of action of disulfiram in the treatment of alcohol dependence. Pharmacopsychiatry 49(4):137-141

71. National Institute for Health and Care Excellence (2011) Alcohol use disorders: diagnosis, assessment and management of harmful drinking and alcohol dependence. National Institute for Health and Care Excellence, London

72. Naudet F, Palpaceur V, Boussageon R (2016) Evaluation in alcohol use disorders - insights from the nalmefene experience. BMCMed 14:119-127

73. O'Malley SS, Jaffe AJ, Chang G (1992) Naltrexone and coping skills therapy for alcohol dependence. A controlled study. Arch Gen Psychiatry 49(11):881-887

74. Oon-Arom A, Likhitsathian S, Srisuraparont M (2019) Efficacy and acceptability of varenicline for alcoholism: a systematic review and meta-analysis of randomized-controlled trials. Drug Alcohol Depend 205:107631

75. Palpacuer C, Laviolle B, Boussageon R et al (2015) Risks and benefits of nalmefene in the treatment of adult alcohol dependence: a systematic literature review and meta-analysis of published and unpublished double-blind randomized controlled trials. PLoS Med 12:e1001924

76. Palpacuer C, Duprez R, Huneau A et al (2018) Pharmacologically controlled drinking in the treatment of alcohol dependence or alcohol use disorders: a systematic review with direct and network meta-analyses on nalmefene, naltrexone, acamprosate, baclofen and topiramate. Addiction 113(2):220-237

77. Pani PP, Trogu E, Pacini M et al (2014) Anticonvulsants for alcohol dependence. Cochrane Database Syst Rev 2:Cd8544

78. Ponizovsky AM, Rosca P, Aronovich E et al (2015) Baclofen as add-on to standrard psychosocial treatment for alcohol dependence: a randomized, double-blind, placebo controlled trial with 1 year follow-up. JSubst Abuse Treat 52:24-30

79. Ragia G, Manopoulos VG (2017) Personalized medicine of alcohol addiction: pharmacogenomics and beyond. Curr Pharm Biotechnol 18:221-230

80. Ray LA, Green RJ, Roche DJO et al (2019) Naltrexone effects on subjective responses to alcohol in the human laboratory: a systematic review and metaanalysis. Addict Biol 24:1138-1152

81. Rehm J, Dawson D, Frick U et al (2014) Burden of disease associated with alcohol use disorders in the United States. Alcohol Clin Exp Res 38(4):1068-1077

82. Reynaud M, Aubin H-J, Trinquet Fet al (2017) A randomized, placebo-controlled study of high-dose baclofen in alcohol-dependent patients-the ALPADIR study. Alcohol Alcohol 52(4):439446

83. Roche DJO, Ray LA (2015) Subjective response as a consideration in the pharmacogenetics of alcoholism treatment. Pharmacogenomics 16:721-726

84. Rolland B, Labreuche J, Duhamel A et al (2015) Baclofen for alcohol dependence: relationships between baclofen and alcohol dosing and the occurrence of major sedation. Eur Neuropsychopharmacol 25:1631-1636

85. Rösner S, Hackl-Herrwerth A, Leucht S et al (2010a) Acamprosate for alcohol dependence. Cochrane Database Syst Rev 9:CD4332

86. Rösner S, Hackl-Herrwerth A, Leucht S et al (2010b) Opioid antagonists for alcohol dependence. Cochrane Database Syst Rev 12:CD1867

87. Rösner S, Grosshans M, Mutschler JH (2014) Disulfiram: Aktuelle Befunde und Wirkmechanismen. Suchtmedizin 16(2):47-52
88. Rösner S, Soyka M (2019) Outcome assessment in trials of pharmacological treatments for alcohol use disorders. CNSDrugs 33:649-657

89. Simpson TL, Saxon AJ, Stappenbeck $C$ et al (2018) Double-blind randomized clinical trial of prazosin for alcohol use disorder. Am J Psychiatry 175(12):1216-1224

90. Sinclair JM, Chambers SE, Shiles CJetal (2016) Safety and tolerability of pharmacological treatment of alcohol dependence: comprehensive review of evidence. Drug Saf 39:627-645

91. Skinner MD, Coudert M, Berlin I et al (2010) Effect of the threat of a disulfiram-ethanol reaction on cue reactivity in alcoholics. Drug Alcohol Depend 112(3):239-246

92. Skinner MD, Lahmek P, Pham H et al (2014) Disulfiram efficacy in the treatment of alcohol dependence: a meta-analysis. PLoSOne 9(2):e87366

93. Soyka M, Müller CA (2017) Pharmacotherapy of alcoholism-an update on approved and offlabel medications. Expert Opin Pharmacother 18(12):1187-1199

94. Soyka M (2016) Nalmefene for the treatment of alcohol use disorders: recent data and clinical potential.ExpertOpin Pharmacother 7(4):619-626

95. Soyka M, Batra A, Heinz A et al (Hrsg) (2019) Suchtmedizin. Elsevier, München

96. Spanagel R, Vengeliene V, Jandeleit B et al (2014) Acamprosate produces its anti-relapse effects via calcium. Neuropsychopharmacology 39(4):783-791

97. Stanciu C, Penders T, Wuensch Ket al (2017) Underutilization of pharmacotherapy for treatment of alcohol use disorders part ll-results from a survey of practices among North Carolina mental health providers and brief review of efficacy of available pharmacotherapies. J Alcohol Drug Depend 5(5):285

98. Stevenson M, Pandor A, Stevens JW et al (2015) Nalmefene for reducing alcohol consumption in people with alcohol dependence: an evidence review group perspective of a NICE single technology appraisal. PharmacoEconomics 33:833-847

99. Tiffany ST, Friedman L, Greenfield SF (2012) Beyond drug use: a systematic consideration of other outcomes in evaluations of treatments for substance use disorders. Addiction 107(4):709-718

100. van den Brink W, Aubin HJ, Bladström A et al (2013) Effiaccy of as-needed nalmefene in alcohol dependent patients with at least a high drinking risk level: results from a subgroup analysis of two randomized controlled 6-month studies. Alcohol Alcohol 48:570-578

101. van den Brink W, Sorensen P, Torup L et al (2014) Long-term efficacy, tolerability and safety of nalmefene as-needed in patients with alcohol dependence: a 1-year, randomized controlled study. J Psychopharmacol 28:733-744

102. van den Brink W, Addolorato G, Aubin H-J et al (2018) Efficacy and safety of sodium oxybate in alcohol-dependent patients with a very high drinking risk level. Addict Biol 23:969-986

103. Vickers-Smith R, Sun J, Charnigo RJ et al (2020) Gabapentin drug misuse signals: a pharmacovig-ilance assessment using the FDA adverse event reporting system. Drug Alcohol Depend 206:107709

104. Volpicelli JR, Alterman Al, Hayashida M et al (1992) Naltrexone in the treatment of alcohol dependence. Arch Gen Psychiatry 49(11):876-880

105. Witkiewitz K, Litten RZ, Leggio L (2019) Advances in the science and treatment of alcohol use disorder. Sci Adv 5(9):eaax4043 